Article

\title{
Application of Exponential Jensen Picture Fuzzy Divergence Measure in Multi-Criteria Group Decision Making
}

\author{
Shouzhen Zeng ${ }^{1,2}$, Shahzaib Asharf ${ }^{3, *(\mathbb{D}}$, Muhammad Arif ${ }^{3(\mathbb{D}}$ and Saleem Abdullah ${ }^{3, *}$ \\ 1 School of Business, Ningbo University, Ningbo 315211, China; zszzx1@163.com \\ School of Managment, Fudan University, Shanghai 200433, China \\ 3 Department of Mathematics, Abdul Wali Khan University, Mardan 23200, Pakistan; \\ marifmaths@awkum.edu.pk \\ * Correspondence: shahzaibashraf@awkum.edu.pk (S.A.); saleemabdullah@awkum.edu.pk (S.A.)
}

Received: 14 January 2019; Accepted: 13 February 2019; Published: 17 February 2019

\begin{abstract}
A divergence measure plays a crucial part in discriminating two probability distributions and drawing inferences constructed on such discrimination. The intention of this study is to propose such a divergence measure based on Jensen inequality and exponential entropy in the settings of probability theory. Further, the idea has been generalized to fuzzy sets to familiarize a novel picture fuzzy divergence measure. Besides proposing the validity, some of its key properties are also deliberated. Finally, two illustrative examples are solved based on the proposed picture fuzzy divergence measure which shows the expediency and effectiveness of the proposed approach.
\end{abstract}

Keywords: exponential Jensen divergence; picture fuzzy divergence measure; decision making

\section{Introduction}

Decision making (DM) means that the optimal alternative is selected from the finite set of alternatives according to the multiple criteria, which can be regarded as cognitive processing. Decision making theory is a very important branch, which is used mostly in human activities. Because the real decision making problems are frequently produced from a complicated environment, the evaluation information is usually fuzzy. In general, the fuzzy information takes two forms: one quantitative and other one is qualitative. The quantitative fuzzy information can be expressed by fuzzy set (FS) [1], intuitionistic fuzzy set (IFS) [2], Pythagorean fuzzy set (PyFS) [3,4], picture fuzzy set (PFS) [5] and so on. FS theory proposed by Zadeh [1] has been used to describe fuzzy quantitative information which contains only a membership degree. Due to its successful applications and some shortcomings, many researchers introduced the extended forms of fuzzy set.

Atanassov [2] defined the notion of intuitionistic fuzzy set. In different areas, intuitionistic fuzzy set theory is applied, but in real life, some situations occur that cannot be handled by IFSs. Voting is a good example, because in voting human opinions involving more types of answers such as: "yes", "abstain", "no" and "refusal". For example, in a democratic election station, the council issues 500 voting papers for a candidate. The results of the voting are divided into four groups accompanied with the number of papers that are "vote for" (251), "abstain" (99), "vote against" (120) and "refusal of voting"(30). Here, group "abstain" means that the voting paper is a white paper rejecting both "agree" and "disagree" for the candidate but still takes the vote, group "refusal of voting" is either invalid voting papers or did not take the vote. The candidate is successful because the number of support papers is over half (i.e., 250). However, at least 5 people said later on in their blogs that they supported the candidate in the last moment because they found that the support number seemed 
larger than the against number. Such kinds of examples (in which the number of abstains is a key factor and the group "refusal of voting" indeed exists) happened in reality and intuitionistic fuzzy set could not handle it. So, Cuong [5,6] defined the extension of fuzzy sets and intuitionistic fuzzy sets, which is picture fuzzy sets. The concept of picture fuzzy set for an element is that, there are three membership degrees namely, positive membership degree, the neutral membership degree, and the negative membership degree, respectively. The picture fuzzy set theory is used in many real life problems such as voting problems, clustering [7], fuzzy inference [8], and decision making [9-15].

In view of these developments, we will present new similarity measures for the PFSs. A similarity measure give the similarity degree of objects i.e., how similar these objects are. The similarity measures developed in [8,16-18] have some limitations and cannot be applied to those problems in which the information occurred in the picture fuzzy environment. To resolve this issue, some novel proposed measures which are generalizations of the similarity measures were developed in [8,16-18] . It is also proved that existing similarity measures become special cases of the developed similarity measures, showing the novelty and diversity of the proposed similarity measures. Applying the new similarity measures, we solved decision making problems and their results are discussed.

The remainder of the study is designed as follows. Section 2 briefly discusses the basic knowledge of extension of fuzzy sets. The novel idea "Picture Fuzzy Divergence Measure" is presented in Section 3. Section 4 makes some discussions on basic properties and Section 5 presents the application of the proposed method. Section 6 discusses the advantages of the proposed work. Conclusions are drawn in Section 7.

\section{Preliminaries}

The article gives a brief discussion on the basic ideas associated with PFS along with their operations and operators. We also discuss more familiarized ideas, which are utilized in the following analysis.

Cuong [5] proposed the idea of picture fuzzy sets and their basic operational rules are as follows

Definition 1. For a set $R$, by a picture fuzzy set in $R$ we mean a structure

$$
\mu=\left\{<p_{\mu}(f), i_{\mu}(f), g_{\mu}(f)>\mid f \in R\right\},
$$

in which $p_{\mu}: p \rightarrow[0,1], i_{\mu}: p \rightarrow[0,1]$ and $g_{\mu}: p \rightarrow[0,1]$ are indicated the positive, neutral and negative grads in $R$. In addition, following condition satisfied by $p_{\mu}, i_{\mu}$ and $g_{\mu}$ is $0 \leq p_{\mu}(f)+i_{\mu}(f)+g_{\mu}(f) \leq 1$; for all $f \in R$. Then $\mu$ is said to be picture fuzzy set in $R$.

Definition 2. Let $\mu_{1}=\left\langle p_{\mu_{1}}, i_{\mu_{1}}, g_{\mu_{1}}\right\rangle$ and $\mu_{2}=\left\langle p_{\mu_{2}}, i_{\mu_{2}}, g_{\mu_{2}}\right\rangle$ be two PFSs in $R$. Then

(1) $\mu_{1} \subseteq \mu_{2}$ if and only if $p_{\mu_{1}} \leq p_{\mu_{2}}, i_{\mu_{1}} \geq i_{\mu_{2}}$ and $g_{\mu_{1}} \geq g_{\mu_{1}}$ for all $f \in R$;

(2) $\mu_{1}=\mu_{2}$ if and only if $\mu_{1} \subseteq \mu_{2}$ and $\mu_{2} \subseteq \mu_{1}$;

(3) $\mu_{1}^{c}=\left\{\left\langle g_{\mu_{1}}(f), i_{\mu_{1}}(f), p_{\mu_{1}}(f)\right\rangle \mid f \in R\right\}$;

(4) $\mu_{1} \cup \mu_{2}=\left\{\begin{array}{c}\left\langle\max \left(p_{\mu_{1}}(f), p_{\mu_{2}}(f)\right), \min \left(i_{\mu_{1}}(f), i_{\mu_{2}}(f)\right), \min \left(g_{\mu_{1}}(f), g_{\mu_{2}}(f)\right)\right\rangle \\ \mid f \in R\end{array}\right\}$;

(5) $\mu_{1} \cap \mu_{2}=\left\{\begin{array}{c}\left\langle\min \left(p_{\mu_{1}}(f), p_{\mu_{2}}(f)\right), \min \left(i_{\mu_{1}}(f), i_{\mu_{2}}(f)\right), \max \left(g_{\mu_{1}}(f), g_{\mu_{2}}(f)\right)\right\rangle \\ \mid f \in R\end{array}\right\}$.

Definition 3. A function $\stackrel{*}{T}:[0,1] \times[0,1] \rightarrow[0,1]$ such that for all $p, i, g \in[0,1]$ is said to be a triangular-norm, if it satisfies the following properties:

(1) $\stackrel{*}{T}(p, i)=\stackrel{*}{T}(i, p)$;

(2) $\stackrel{*}{T}(p, \stackrel{*}{T}(i, g)=\stackrel{*}{T}(\stackrel{*}{T}(p, i), g)$; 
(3) $\stackrel{*}{T}(p, i) \leq \stackrel{*}{T}(p, g)$, whenever $i \leq g$;

(4) $\stackrel{*}{T}(1, p)=p$.

Definition 4. A function $\stackrel{*}{S}:[0,1] \times[0,1] \rightarrow[0,1]$ such that for all $p, i, g \in[0,1]$ is said to be a triangular-conorm, if it satisfies the following properties:

(1) $\stackrel{*}{S}(p, i)=\stackrel{*}{S}(i, p)$;

(2) $\stackrel{*}{S}(p, \stackrel{*}{S}(i, g)=\stackrel{*}{S}(\stackrel{*}{S}(p, i), g)$;

(3) $\stackrel{*}{S}(p, i) \leq \stackrel{*}{S}(p, g)$, whenever $i \leq g$;

(4) $\stackrel{*}{S}(0, p)=p$.

Joshi and Kumar [17] discussed the basic norms functions which are defined as

Definition 5. Some basic norms are the following:

(1) Minimum traingular-norm:

$$
\stackrel{*}{T}_{\min }(p, i)=\min (p, i)
$$

(2) Product traingular-norm:

$$
\stackrel{*}{T}_{\text {prod }}(p, i)=(p \cdot i)
$$

(3) Lukasiewicz traingular-norm:

$$
\stackrel{*}{T}_{\text {luka }}(p, i)=\max (p+i-1,0)
$$

(4) Drastic product traingular-norm:

$$
\stackrel{*}{T}_{\text {luka }}(p, i)=\left\{\begin{array}{cc}
0 & \text { if } p \vee i \in 1 \\
\min (p, i) & \text { otherwise. }
\end{array}\right.
$$

Similarly, we define the four basic triangular-conorms.

\section{Picture Fuzzy Divergence Measure}

History: Suppose that $\Lambda_{n}=\left\{G=\left(g_{1}, g_{2}, \ldots, g_{n}\right) ; g_{p} \geq 0, \sum_{p=1}^{n} g_{p}=1\right\}, n \geq 2$ are the complete probability distributions set. Shannon entropy of a set $G$ is follows as:

$$
H(G)=-\sum_{p=1}^{n} g_{p} \log \left(g_{p}\right)
$$

The concavity of $H(G)$ gives us a decomposition of the overall diversity in a mixed distribution $\left(\frac{G+D}{2}\right)$ is follows;

$$
H\left(\frac{G+D}{2}\right)=\frac{1}{2}\left(H(G)+H(D)+\frac{1}{2} J_{n}(G, D)\right.
$$

The first part of Equation (2), that is $\left(\frac{H(G)+H(D)}{2}\right)$ denotes the average diversity within distributions, where the second component, that is

$$
J_{n}(G, D)=(-H(G)-H(D))-2\left(-H\left(\frac{G+D}{2}\right)\right)
$$


is called the Jensen difference arising out of the convex function $-H(G)$, which is nonnegative and disappears if and only if $G=D$ and this gives a natural measure of divergence between the distributions $G$ and $D$. It is notable that $J_{n}(G, D)$ assumed as a function of $(G, D)$, is convex, which meets the intuitive condition that the average divergence between $(G, D),(C, L)$ and $(E, R)$ is not less than that between their convex combination $\mu(G, D)+\eta(C, L)+v(E, R)$, where $\mu, \eta, v \geq 0$ and $\mu+\eta+v=1$. The convexity of divergence measure $J_{n}(A, B)$ is an additional attractive feature of the Shannon entropy $H(G)$ as a measure of diversity of a distribution. The application of measure of diversity is discussed in $[19,20]$.

In this study, we assume that the Jensen difference Equation (3) arising from a generalized class of entropy functions including the exponential entropy due to Pal and Pal [21], which is called an exponential J-divergence, and examine its convexity. The convexity of J-divergence Equation (3) is proved, based on the exponential entropy given by

$$
H(G)=\sum_{p=1}^{n} g_{p}\left(e^{1-g_{p}}-1\right) ; G \in \Lambda_{n}
$$

The author claims that Equation (4) has some advantages over Equation (1) specifically in image processing. Another claim is that entropy Equation (4) has a fixed upper bound, that is, for a uniform distribution $\left(\frac{1}{n}, \frac{1}{n}, \ldots, \frac{1}{n}\right) ; \lim _{n \rightarrow \infty} H(G)=e-1$ as compared to infinite limit $($ as $n \rightarrow \infty)$ in the case of Equation (1).

For any two probability distributions $G, D \in \Lambda_{n}$ with respect to the weights $\sigma_{1}, \sigma_{2}=1$, Lin [22] defined Jensen-Shannon divergence as:

$$
J S_{\mu}(G, D)=H\left(\sigma_{1} G+\sigma_{2} D\right)-\sigma_{1} H(G)-\sigma_{2} H(D)
$$

Since Equation (1) denotes a concave function, according to the Jensen inequality, when $G=D$, then $J S_{\mu}(G, D)$ is nonnegative and is equal to zero. Based on the concept of Jensen-Shannon divergence (Lin [22]), corresponding to Equation (4), for any two probability distributions $G, D \in \Lambda_{n}$ with $\sigma_{1}$ and $\sigma_{2}$ as their respective weights satisfying we define a new divergence measure as:

$$
\widehat{P_{F D}}(G, D)=\sum_{p=1}^{n}\left[\begin{array}{c}
\left(\sigma_{1} g_{p}+\sigma_{2} d_{p}\right) e^{1-\left(\sigma_{1} g_{p}+\sigma_{2} d_{p}\right)}+ \\
\left(\sigma_{1} d_{p}+\sigma_{2} g_{p}\right) e^{1-\left(\sigma_{1} d_{p}+\sigma_{2} g_{p}\right)}- \\
\left(g_{p} e^{1-g_{p}}\right)-\left(d_{p} e^{1-d_{p}}\right)
\end{array}\right]
$$

Definition 6. The Hessian function $j$ of any two variables $g$ and $d$ is

$$
\operatorname{Hessian}(j)=\left[\begin{array}{ll}
\frac{\delta^{2} j}{\delta \delta^{2}} & \frac{\delta^{2} j}{\delta g \delta d} \\
\frac{\delta^{2} j}{\delta g^{2} \delta d} & \frac{\delta^{2} j}{\delta d^{2}}
\end{array}\right]
$$

A function $j$ is convex at any point in its domain if Hessian $(j)$ is positive semi-definite and concave if Hessian $(j)$ is negative semi-definite at that point.

To justify the existence of new divergence measure, which is:

$$
\widehat{P_{F D}}(G, D)=\sum_{p=1}^{n}\left[\begin{array}{c}
\left(\sigma_{1} g_{p}+\sigma_{2} d_{p}\right) e^{1-\left(\sigma_{1} g_{p}+\sigma_{2} d_{p}\right)}+ \\
\left(\sigma_{1} d_{p}+\sigma_{2} g_{p}\right) e^{1-\left(\sigma_{1} d_{p}+\sigma_{2} g_{p}\right)}- \\
\left(g_{p} e^{1-g_{p}}\right)-\left(d_{p} e^{1-d_{p}}\right)
\end{array}\right]
$$

we have to prove that proposed divergence measure $\widehat{P_{F D}}(G, D)$ satisfies the following properties.

Some major properties of proposed divergence measure are the following:

(1) $\widehat{P_{F D}}(G, D) \geq 0$ with equality when $G=D$; 
(2) $\widehat{P_{F D}}(G, D)$ is a convex function of $G$ and $D$.

Proof. We have to show that $\widehat{P_{F D}}(G, D) \geq 0$. For this from Jensen's inequality, we have

$$
H\left(\sigma_{1} G+\sigma_{2} D\right) \geq \sigma_{1} H(G)+\sigma_{2} H(D)
$$

This implies

$$
\sum_{p=1}^{n}\left(\sigma_{1} g_{p}+\sigma_{2} d_{p}\right) e^{1-\left(\sigma_{1} g_{p}+\sigma_{2} d_{p}\right)} \geq \sigma_{1} \sum_{p=1}^{n} g_{p} e^{1-g_{p}}+\sigma_{2} \sum_{p=1}^{n} d_{p} e^{1-d_{p}}
$$

Now, we have to show the convexity of function $j$, where

$$
j(g, d)=\left(\sigma_{1} g+\sigma_{2} d\right) e^{1-\left(\sigma_{1} g+\sigma_{2} d\right)}-\sigma_{1} g e^{1-g}-\sigma_{2} d e^{1-d} .
$$

Taking partial differential of the above example with respect to $g$ and $d$, we obtain

$$
\frac{\delta j}{\delta g}=\sigma_{1} e^{\left(1-\sigma_{1} g-\sigma_{2} d\right)}\left(1-\sigma_{1} g-\sigma_{2} d\right)+\sigma_{1} e^{1-g}(g-1) ;
$$

and

$$
\frac{\delta j}{\delta d}=\sigma_{1} e^{\left(1-\sigma_{2} g-\sigma_{1} d\right)}\left(1-\sigma_{2} g-\sigma_{1} d\right)+e^{1-d}(d-1) ;
$$

Put $\frac{\sigma f}{\sigma a}=0$ and $\frac{\sigma f}{\sigma b}=0$ to find the stationary point.This gives $g=d$ as a stationary point. Now, computing the Hessian of $j$ at $g=d$ and using $\sigma_{1}+\sigma_{2}=1$, we obtain

$$
\operatorname{Hessian}(j)=e^{1-g}(2-g) \sigma_{1} \sigma_{2}\left[\begin{array}{ll}
1 & -1 \\
-1 & 1
\end{array}\right]
$$

which is positive semi-definite. This confirms the convex character of $f$.

Now, we propose the novel divergence measure based on the Jensen's divergence as follows:

Definition 7. Let the universe set $R, \mu_{1}=\left\langle p_{\mu_{1}}, i_{\mu_{1}}, g_{\mu_{1}}\right\rangle, \mu_{2}=\left\langle p_{\mu_{2}}, i_{\mu_{2}}, g_{\mu_{2}}\right\rangle$ be two PFNs in $R$ and $\sigma_{1}>0, \sigma_{2}>0, \sigma_{1}+\sigma_{2}=1$. Picture fuzzy divergence measure $\widehat{P_{F D}}\left(\mu_{1}, \mu_{2}\right)$ is define as,

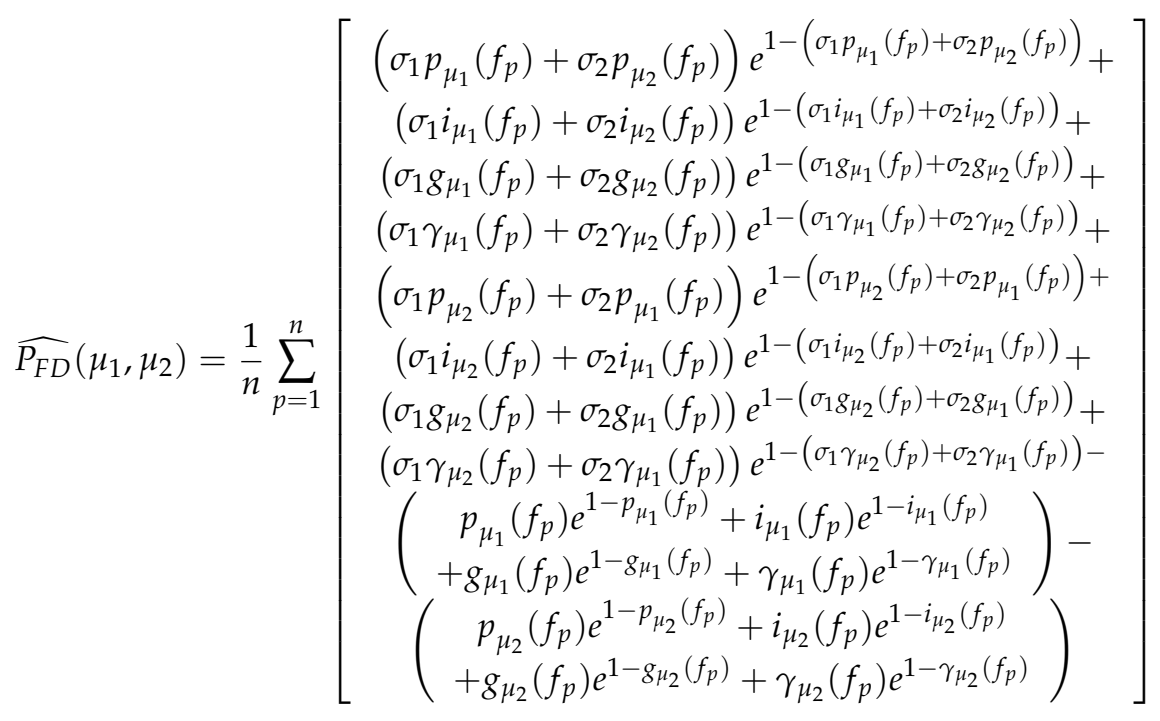

\section{Properties of Picture Fuzzy Divergence Measure}

Picture fuzzy divergence measure for PFNs satisfies the following defined properties as; 
Theorem 1. Let the universe set $R, \mu_{1}=\left\langle p_{\mu_{1}}, i_{\mu_{1}}, g_{\mu_{1}}\right\rangle, \mu_{2}=\left\langle p_{\mu_{2}}, i_{\mu_{2}}, g_{\mu_{2}}\right\rangle$ and $\mu_{3}=\left\langle p_{\mu_{3}}, i_{\mu_{3}}, g_{\mu_{3}}\right\rangle$ be any three PFNs in $R$. Then

(1) $\widehat{P_{F D}}\left(\mu_{1}, \mu_{2}\right)=\widehat{P_{F D}}\left(\mu_{2}, \mu_{1}\right)$

(2) $\widehat{P_{F D}}\left(\mu_{1}, \mu_{2}\right)=0$ iff $\mu_{1}=\mu_{2}$;

(3) $\widehat{P_{F D}}\left(\mu_{1}, \mu_{1}^{c}\right)=0$ iff $p_{\mu_{1}}^{c}\left(f_{p}\right)=1-p_{\mu_{1}}\left(f_{p}\right)$ for all $f_{p} \in R$;

(4) $\widehat{P_{F D}}\left(\mu_{1}, \mu_{1} \cup \mu_{2}\right)=\widehat{P_{F D}}\left(\mu_{1} \cap \mu_{2}, \mu_{2}\right) \leq \widehat{P_{F D}}\left(\mu_{1}, \mu_{2}\right)$;

(5) $\widehat{P_{F D}}\left(\mu_{1} \cup \mu_{2}, \mu_{1} \cap \mu_{2}\right)=\widehat{P_{F D}}\left(\mu_{1} \cap \mu_{2}, \mu_{1} \cup \mu_{2}\right)=\widehat{P_{F D}}\left(\mu_{1}, \mu_{2}\right)$;

(6) $\widehat{P_{F D}}\left(\mu_{1} \cap \mu_{2}, \mu_{1} \cup \mu_{2}\right)=\widehat{P_{F D}}\left(\mu_{2}, \mu_{1}\right)$

(7) $\widehat{P_{F D}}\left(\mu_{1}, \mu_{1} \cup \mu_{2}\right)+\widehat{P_{F D}}\left(\mu_{1}, \mu_{1} \cap \mu_{2}\right)=\widehat{P_{F D}}\left(\mu_{1}, \mu_{2}\right)$;

(8) $\widehat{P_{F D}}\left(\mu_{2}, \mu_{1} \cup \mu_{2}\right)+\widehat{P_{F D}}\left(\mu_{2}, \mu_{1} \cap \mu_{2}\right)=\widehat{P_{F D}}\left(\mu_{2}, \mu_{1}\right)$;

(9) $\widehat{P_{F D}}\left(\mu_{1} \cup \mu_{2}, \mu_{3}\right) \leq \widehat{P_{F D}}\left(\mu_{1}, \mu_{3}\right)+\widehat{P_{F D}}\left(\mu_{2}, \mu_{3}\right)$;

(10) $\widehat{P_{F D}}\left(\mu_{1} \cap \mu_{2}, \mu_{3}\right) \leq \widehat{P_{F D}}\left(\mu_{1}, \mu_{3}\right)+\widehat{P_{F D}}\left(\mu_{2}, \mu_{3}\right)$;

(11) $\widehat{P_{F D}}\left(\mu_{1} \cup \mu_{2}, \mu_{3}\right)+\widehat{P_{F D}}\left(\mu_{1} \cap \mu_{2}, \mu_{3}\right)=\widehat{P_{F D}}\left(\mu_{1}, \mu_{3}\right)+\widehat{P_{F D}}\left(\mu_{2}, \mu_{3}\right)$;

(12) $\widehat{P_{F D}}\left(\mu_{1}, \mu_{2}\right)=\widehat{P_{F D}}\left(\mu_{1}^{c}, \mu_{2}^{c}\right)$;

(13) $\widehat{P_{F D}}\left(\mu_{1}, \mu_{2}^{c}\right)=\widehat{P_{F D}}\left(\mu_{1}^{c}, \mu_{2}\right)$;

(14) $\widehat{P_{F D}}\left(\mu_{1}, \mu_{2}\right)+\widehat{P_{F D}}\left(\mu_{1}^{c}, \mu_{2}\right)=\widehat{P_{F D}}\left(\mu_{1}^{c}, \mu_{2}^{c}\right)+\widehat{P_{F D}}\left(\mu_{1}, \mu_{2}^{c}\right)$

where $\mu_{1}^{c}$ and $\mu_{2}^{c}$ represent the compliment of the PFSs $\mu_{1}$ and $\mu_{2}$, respectively.

Proof. Suppose that $R$ bifurcate into two parts $f$ and $R_{2}$, such that

$$
f=\left\{f_{p} \in R \mid \mu_{1}\left(f_{p}\right) \subseteq \mu_{2}\left(f_{p}\right)\right\} \text { and } R_{2}=\left\{f_{p} \in R \mid \mu_{1}\left(f_{p}\right) \supseteq \mu_{2}\left(f_{p}\right)\right\}
$$

From (7), for all $f_{p} \in f$,

$$
p_{\mu_{1}}\left(f_{p}\right) \leq p_{\mu_{2}}\left(f_{p}\right), i_{\mu_{1}}\left(f_{p}\right) \geq i_{\mu_{2}}\left(f_{p}\right) \text { and } g_{\mu_{1}}\left(f_{p}\right) \geq g_{\mu_{2}}\left(f_{p}\right) ;
$$

and for all

$$
f_{p} \in R_{2}, p_{\mu_{1}}\left(f_{p}\right) \geq p_{\mu_{2}}\left(f_{p}\right), i_{\mu_{1}}\left(f_{p}\right) \leq i_{\mu_{2}}\left(f_{p}\right) \text { and } g_{\mu_{1}}\left(f_{p}\right) \leq g_{\mu_{2}}\left(f_{p}\right) ;
$$

1. Properties (1)-(3) are proved directly from the Definition 5

2. Property (4) as follows. Since

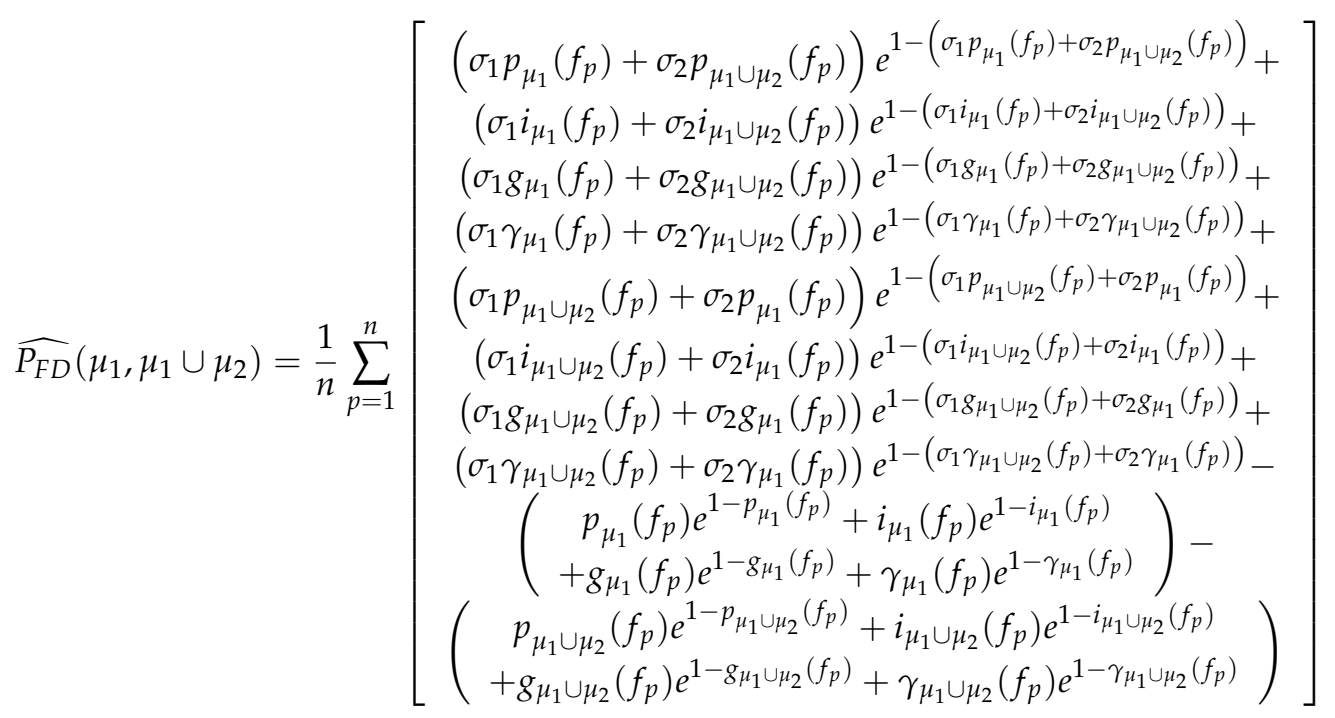

This implies 


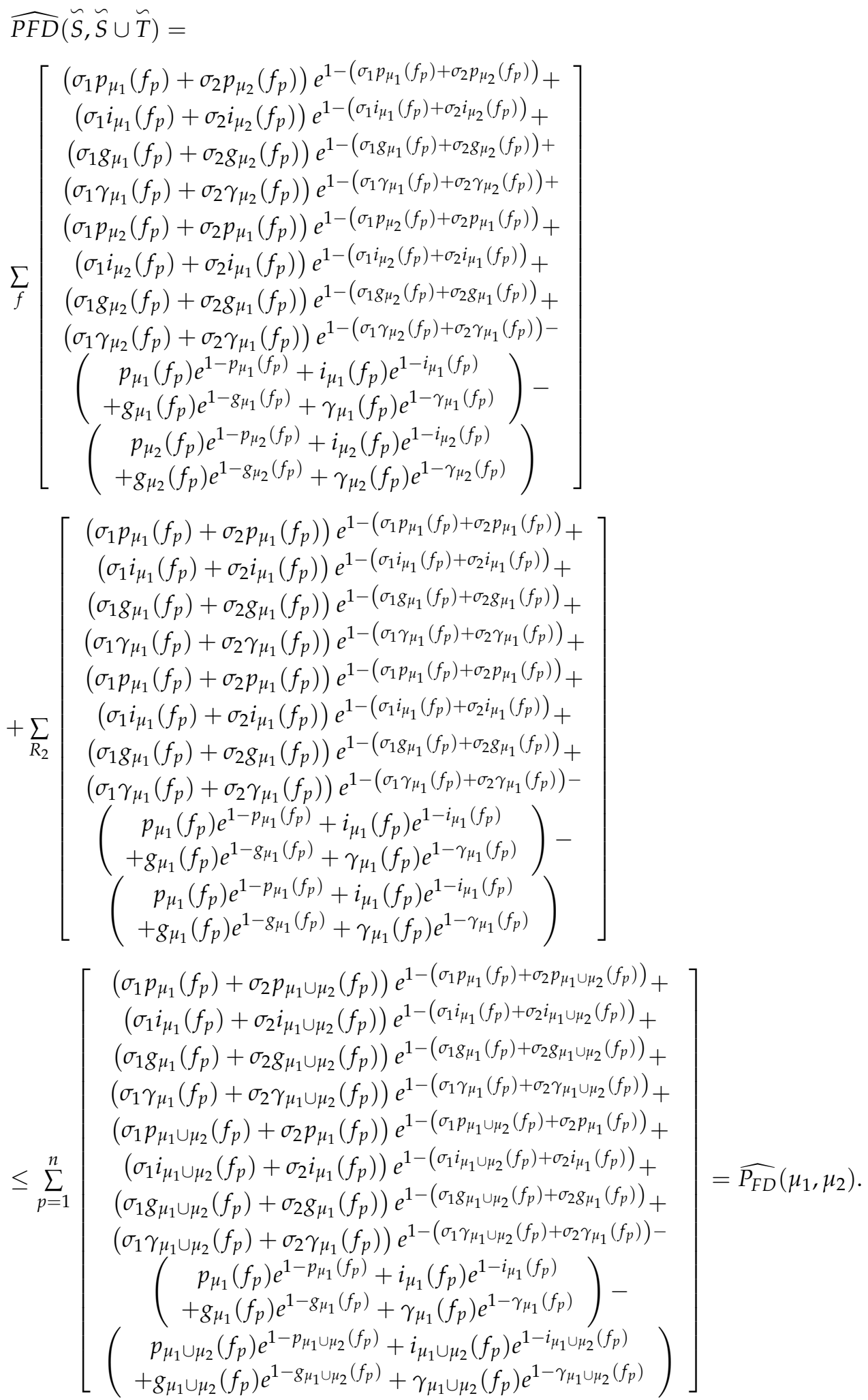

Similarly, we can prove that $\widehat{P_{F D}}\left(\mu_{1} \cap \mu_{2}, \mu_{2}\right) \leq \widehat{P_{F D}}\left(\mu_{1}, \mu_{2}\right)$.

\section{From the property (5),}


we have

$$
\begin{aligned}
\widehat{P_{F D}}\left(\mu_{1} \cup \mu_{2}, \mu_{1} \cap \mu_{2}\right) & =\sum_{f} \widehat{P_{F D}}\left(\mu_{2}, \mu_{1}\right)+\sum_{R_{2}} \widehat{P_{F D}}\left(\mu_{1}, \mu_{2}\right) \\
& =\widehat{P_{F D}}\left(\mu_{1}, \mu_{2}\right),
\end{aligned}
$$

Similarly, we can prove that

$$
\begin{aligned}
\widehat{P_{F D}}\left(\mu_{1} \cap \mu_{2}, \mu_{1} \cup \mu_{2}\right) & =\sum_{f} \widehat{P_{F D}}\left(\mu_{2}, \mu_{1}\right)+\sum_{R_{2}} \widehat{P_{F D}}\left(\mu_{1}, \mu_{2}\right) \\
& =\widehat{P_{F D}}\left(\mu_{1}, \mu_{2}\right) .
\end{aligned}
$$

4. Proof of this property (6) is follows as the proof of properties (5) \& (1).

5. Proof of this property (7)

$$
\begin{aligned}
& \widehat{P_{F D}}\left(\mu_{1}, \mu_{1} \cup \mu_{2}\right)+\widehat{P_{F D}}\left(\mu_{1}, \mu_{1} \cap \mu_{2}\right) \\
= & \sum_{f} \widehat{P_{F D}}\left(\mu_{1}, \mu_{2}\right)+\sum_{R_{2}} \widehat{P_{F D}}\left(\mu_{1}, \mu_{1}\right) \\
& +\sum_{f} \widehat{P_{F D}}\left(\mu_{1}, \mu_{1}\right)+\sum_{R_{2}} \widehat{P_{F D}}\left(\mu_{1}, \mu_{2}\right) \\
= & \widehat{P_{F D}}\left(\mu_{1}, \mu_{2}\right) .
\end{aligned}
$$

6. Proof of this property (8) follows as the proof of property (7).

7. Proof of this property (9), consider $\widehat{P_{F D}}\left(\mu_{1}, \mu_{3}\right)+\widehat{P_{F D}}\left(\mu_{2}, \mu_{3}\right)-\widehat{P_{F D}}\left(\mu_{1} \cup \mu_{2}, \mu_{3}\right)$

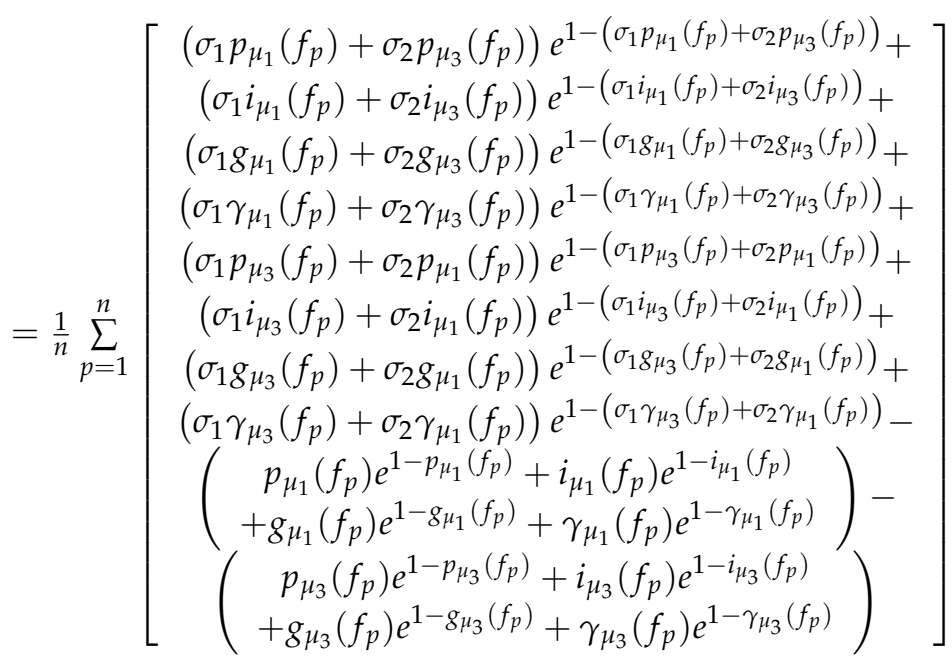




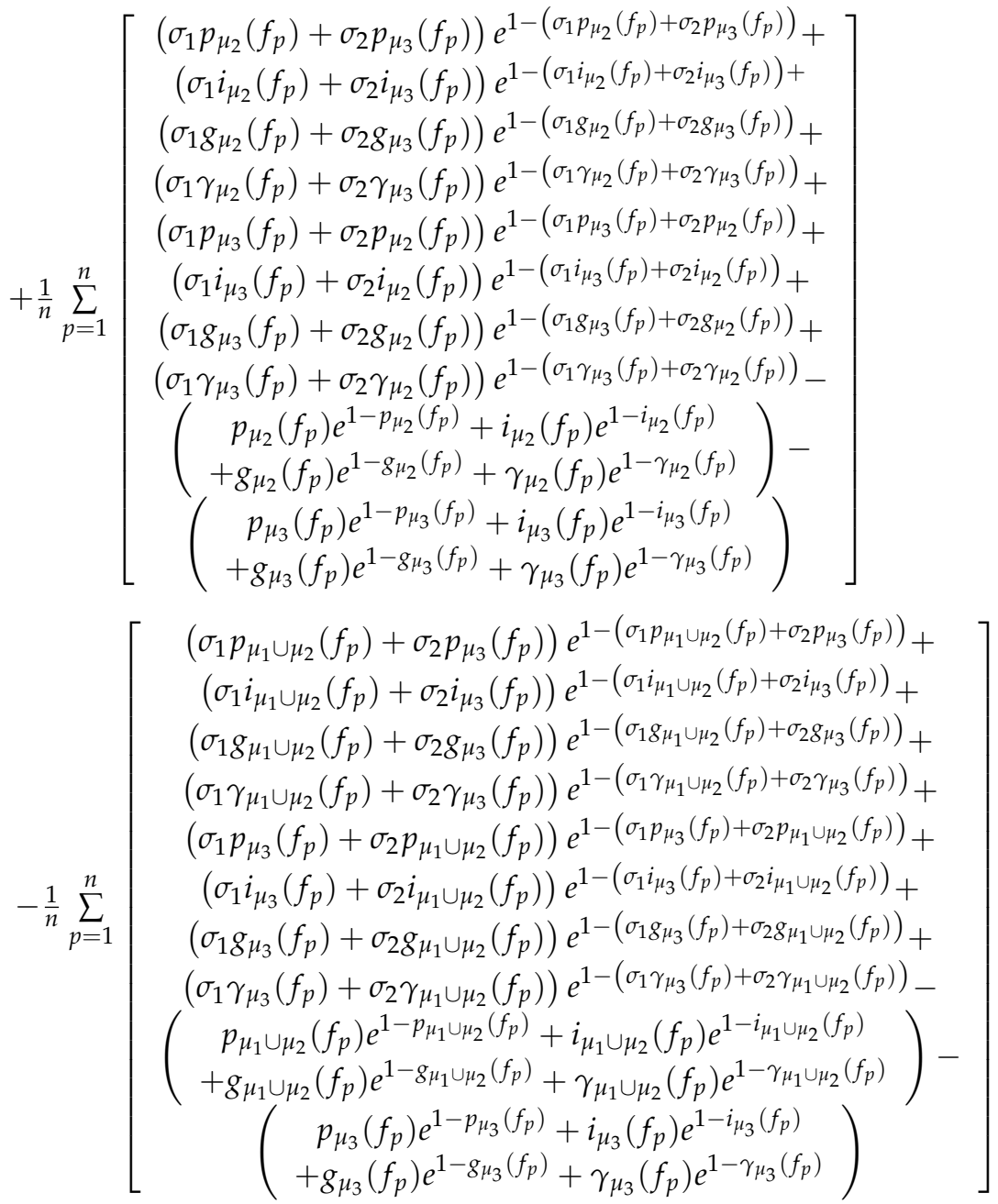

This implies $\widehat{P_{F D}}\left(\mu_{1}, \mu_{3}\right)+\widehat{P_{F D}}\left(\mu_{2}, \mu_{3}\right)-\widehat{P_{F D}}\left(\mu_{1} \cup \mu_{2}, \mu_{3}\right)$

$$
\begin{aligned}
& \begin{array}{c}
\left(\sigma_{1} p_{\mu_{1}}\left(f_{p}\right)+\sigma_{2} p_{\mu_{3}}\left(f_{p}\right)\right) e^{1-\left(\sigma_{1} p_{\mu_{1}}\left(f_{p}\right)+\sigma_{2} p_{\mu_{3}}\left(f_{p}\right)\right)}+ \\
\left(\sigma_{1} i_{\mu_{1}}\left(f_{p}\right)+\sigma_{2} i_{\mu_{3}}\left(f_{p}\right)\right) e^{1-\left(\sigma_{1} i_{\mu_{1}}\left(f_{p}\right)+\sigma_{2} i_{\mu_{3}}\left(f_{p}\right)\right)}+
\end{array} \\
& \left(\sigma_{1} g_{\mu_{1}}\left(f_{p}\right)+\sigma_{2} g_{\mu_{3}}\left(f_{p}\right)\right) e^{1-\left(\sigma_{1} g_{\mu_{1}}\left(f_{p}\right)+\sigma_{2} g_{\mu_{3}}\left(f_{p}\right)\right)}+ \\
& \left(\sigma_{1} \gamma_{\mu_{1}}\left(f_{p}\right)+\sigma_{2} \gamma_{\mu_{3}}\left(f_{p}\right)\right) e^{1-\left(\sigma_{1} \gamma_{\mu_{1}}\left(f_{p}\right)+\sigma_{2} \gamma_{\mu_{3}}\left(f_{p}\right)\right)}+ \\
& \left(\sigma_{1} p_{\mu_{3}}\left(f_{p}\right)+\sigma_{2} p_{\mu_{1}}\left(f_{p}\right)\right) e^{1-\left(\sigma_{1} p_{\mu_{3}}\left(f_{p}\right)+\sigma_{2} p_{\mu_{1}}\left(f_{p}\right)\right)}+ \\
& =\frac{1}{n} \sum_{p=1}^{n} \\
& \left(\sigma_{1} i_{\mu_{3}}\left(f_{p}\right)+\sigma_{2} i_{\mu_{1}}\left(f_{p}\right)\right) e^{1-\left(\sigma_{1} i_{\mu_{3}}\left(f_{p}\right)+\sigma_{2} i_{\mu_{1}}\left(f_{p}\right)\right)}+ \\
& \left(\sigma_{1} g_{\mu_{3}}\left(f_{p}\right)+\sigma_{2} g_{\mu_{1}}\left(f_{p}\right)\right) e^{1-\left(\sigma_{1} g_{\mu_{3}}\left(f_{p}\right)+\sigma_{2} g_{\mu_{1}}\left(f_{p}\right)\right)}+ \\
& \left(\sigma_{1} \gamma_{\mu_{3}}\left(f_{p}\right)+\sigma_{2} \gamma_{\mu_{1}}\left(f_{p}\right)\right) e^{1-\left(\sigma_{1} \gamma_{\mu_{3}}\left(f_{p}\right)+\sigma_{2} \gamma_{\mu_{1}}\left(f_{p}\right)\right)_{-}} \\
& \left(\begin{array}{c}
p_{\mu_{1}}\left(f_{p}\right) e^{1-p_{\mu_{1}}\left(f_{p}\right)}+i_{\mu_{1}}\left(f_{p}\right) e^{1-i_{\mu_{1}}\left(f_{p}\right)} \\
+g_{\mu_{1}}\left(f_{p}\right) e^{1-g_{\mu_{1}}\left(f_{p}\right)}+\gamma_{\mu_{1}}\left(f_{p}\right) e^{1-\gamma_{\mu_{1}}\left(f_{p}\right)}
\end{array}\right)- \\
& \left(\begin{array}{c}
p_{\mu_{3}}\left(f_{p}\right) e^{1-p_{\mu_{3}}\left(f_{p}\right)}+i_{\mu_{3}}\left(f_{p}\right) e^{1-i_{\mu_{3}}\left(f_{p}\right)} \\
+g_{\mu_{3}}\left(f_{p}\right) e^{1-g_{\mu_{3}}\left(f_{p}\right)}+\gamma_{\mu_{3}}\left(f_{p}\right) e^{1-\gamma_{\mu_{3}}\left(f_{p}\right)}
\end{array}\right)
\end{aligned}
$$




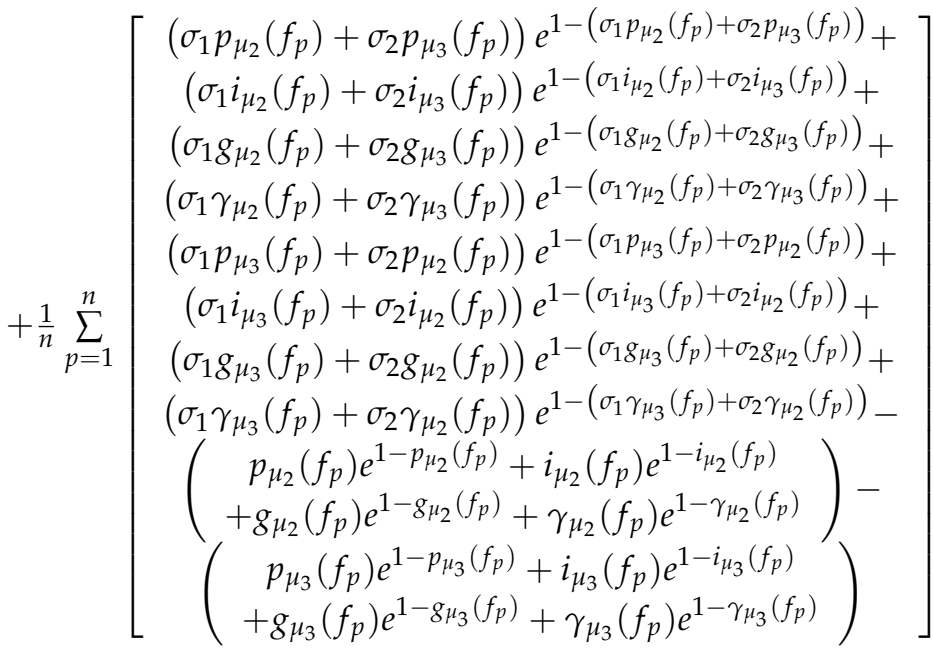

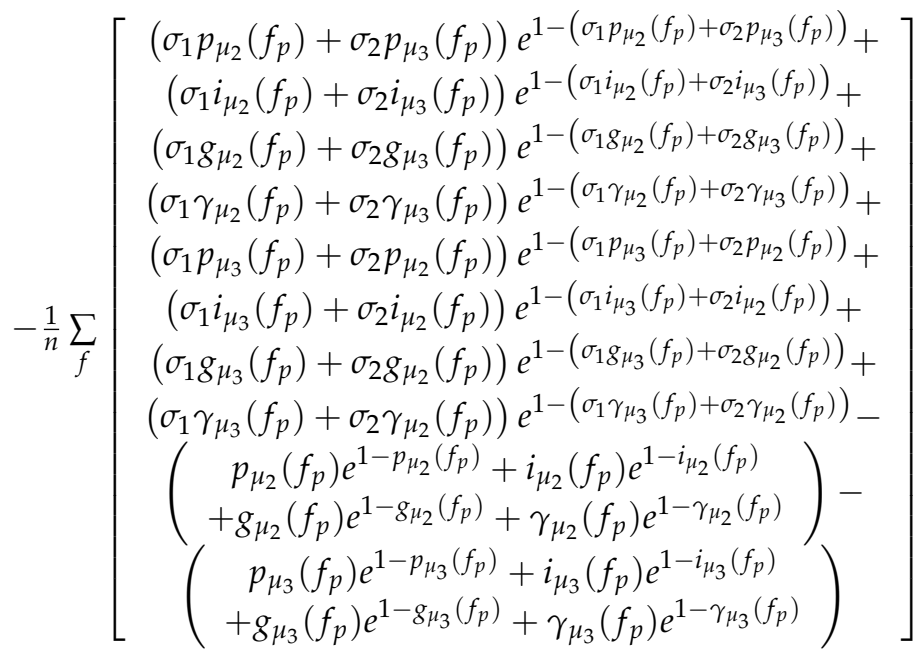

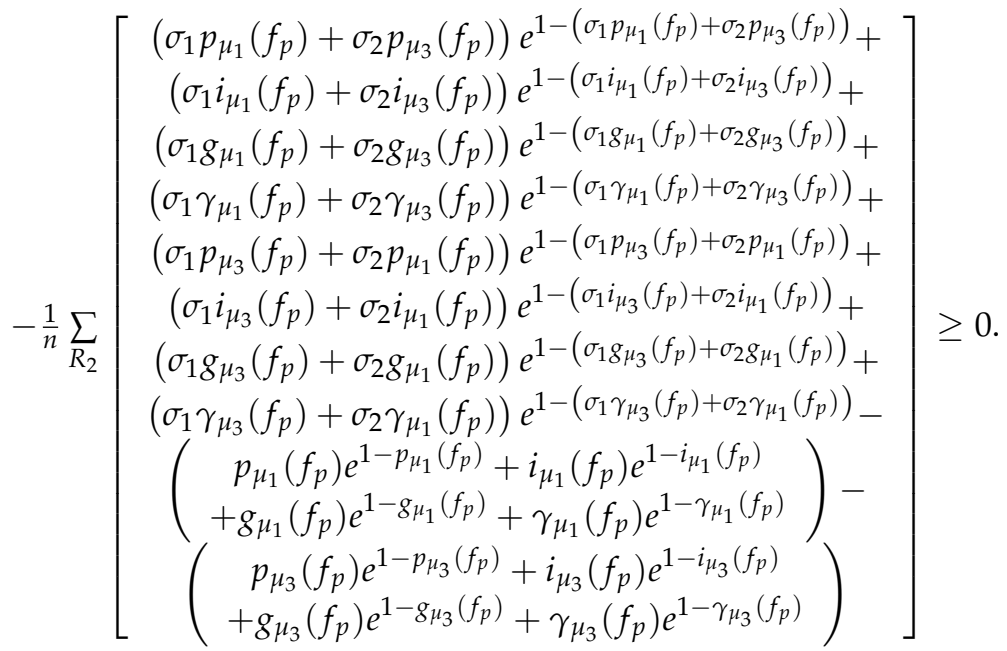

Therefore, $\widehat{P_{F D}}\left(\mu_{1} \cup \mu_{2}, \mu_{3}\right) \leq \widehat{P_{F D}}\left(\mu_{1}, \mu_{3}\right)+\widehat{P_{F D}}\left(\mu_{2}, \mu_{3}\right)$.

8. Proof of this property (10) is follows as the proof of property (9).

9. Proof of this property (11), consider $\widehat{P_{F D}}\left(\mu_{1} \cup \mu_{2}, \mu_{3}\right)+\widehat{P_{F D}}\left(\mu_{1} \cap \mu_{2}, \mu_{3}\right)$ 


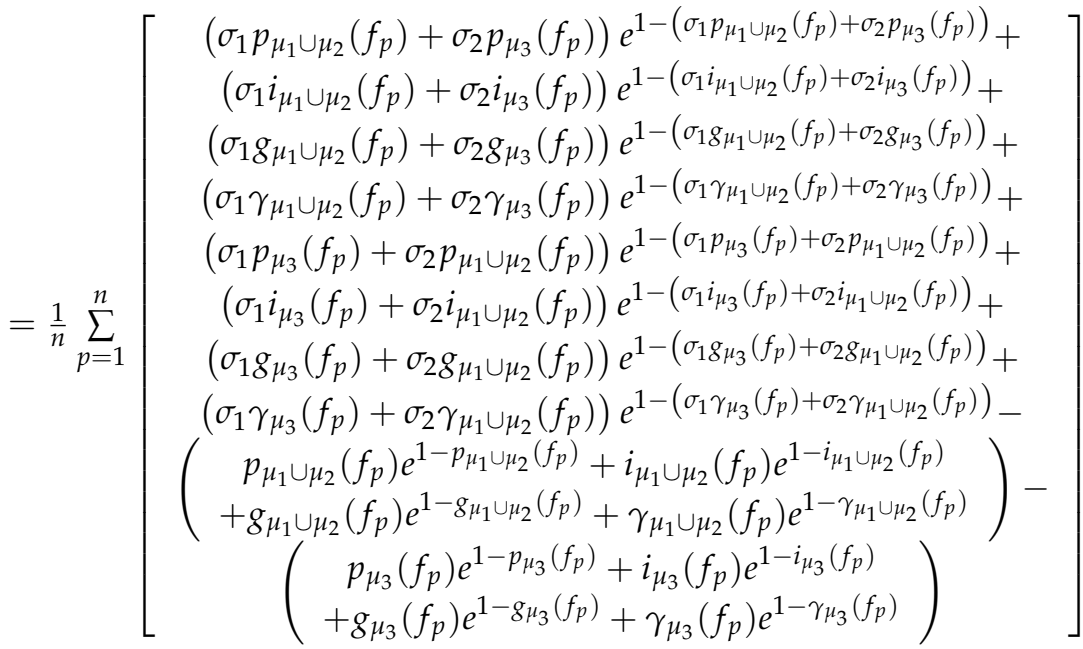

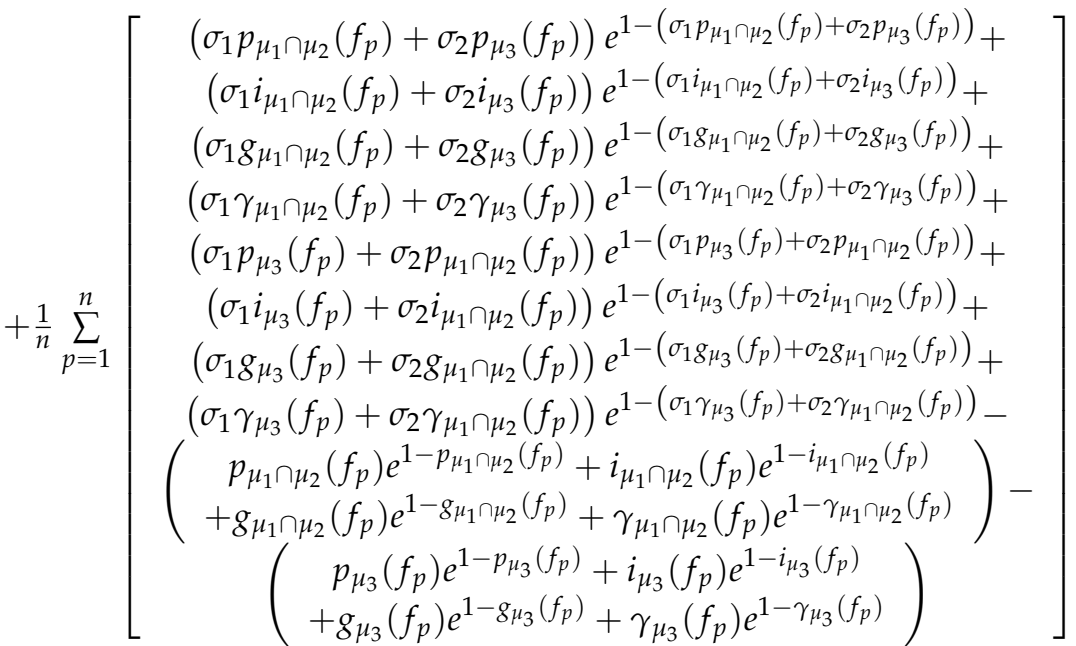

Using (10), we obtain

$$
\begin{aligned}
= & \sum_{f} \widehat{P_{F D}}\left(\mu_{2}, \mu_{3}\right)+\sum_{R_{2}} \widehat{P_{F D}}\left(\mu_{1}, \mu_{3}\right) \\
& +\sum_{f} \widehat{P_{F D}}\left(\mu_{1}, \mu_{3}\right)+\sum_{R_{2}} \widehat{P_{F D}}\left(\mu_{2}, \mu_{3}\right) \\
= & \widehat{P_{F D}}\left(\mu_{1}, \mu_{3}\right)+\widehat{P_{F D}}\left(\mu_{2}, \mu_{3}\right) .
\end{aligned}
$$

10. Proof of properties (12), (13) and (14) follows directly from Definition 5.

\section{Applications of Proposed Picture Fuzzy Divergence Measure in MADM}

In this section, the picture fuzzy divergence measure between picture fuzzy sets is applied to the medical diagnosis and Pattern Recognition.

\subsection{Divergence Measure for Medical Diagnosis}

Assume that a set of diagnoses $G=\left\{G_{1}\right.$ (Viral fever), $G_{2}$ (Malaria), $G_{3}$ (Typhoid), $G_{4}$ (Stomach Problem), $G_{5}$ (Chest Problem) $\}$ and the set of symptoms are $S=\left\{s_{1}\right.$ (Temperature), $s_{2}$ (Head Ache), $s_{3}$ (Stomache Pain), $s_{4}($ Cough $), s_{5}$ (Chest Pain) $\}$. Suppose that the patients, with respect to all symptoms, can be depicted by the following PFS synthetic information: 


$$
\begin{aligned}
& P_{1}=\left\{\begin{array}{c}
s_{1}(\langle 0.6,0.1,0.2,0.1\rangle), s_{2}(\langle 0.3,0.2,0.4,0.1\rangle), s_{3}(\langle 0.4,0.2,0.3,0.1\rangle), \\
s_{4}(\langle 0.7,0.0,0.1,0.2\rangle), s_{5}(\langle 0.4,0.2,0.2,0.2\rangle)
\end{array}\right\} \\
& P_{2}=\left\{\begin{array}{c}
s_{1}(\langle 0.5,0.3,0.1,0.1\rangle), s_{2}(\langle 0.3,0.4,0.3,0.1\rangle), s_{3}(\langle 0.8,0.1,0.1,0.0\rangle), \\
s_{4}(\langle 0.3,0.1,0.5,0.1\rangle), s_{5}(\langle 0.3,0.4,0.2,0.1\rangle)
\end{array}\right\} \\
& P_{3}=\left\{\begin{array}{c}
s_{1}(\langle 0.1,0.1,0.6,0.2\rangle), s_{2}(\langle 0.3,0.1,0.5,0.1\rangle), s_{3}(\langle 0.2,0.2,0.4,0.2\rangle), \\
s_{4}(\langle 0.4,0.4,0.1,0.1\rangle), s_{5}(\langle 0.3,0.2,0.4,0.1\rangle)
\end{array}\right\}
\end{aligned}
$$

Then each diagnoses $G_{i}(i=1,2,3,4,5)$ can be viewed as PFSs with respect to all the symptoms as follows:

$$
\begin{aligned}
& G_{1}=\left\{\begin{array}{c}
s_{1}(\langle 0.4,0.1,0.3,0.2\rangle), s_{2}(\langle 0.5,0.2,0.3,0.0\rangle), s_{3}(\langle 0.4,0.0,0.3,0.3\rangle), \\
s_{4}(\langle 0.7,0.2,0.0,0.1\rangle), s_{5}(\langle 0.6,0.1,0.1,0.2\rangle)
\end{array}\right\} \\
& G_{2}=\left\{\begin{array}{c}
s_{1}(\langle 0.7,0.1,0.1,0.1\rangle), s_{2}(\langle 0.2,0.4,0.3,0.1\rangle), s_{3}(\langle 0.2,0.5,0.1,0.2\rangle), \\
s_{4}(\langle 0.1,0.2,0.5,0.2\rangle), s_{5}(\langle 0.3,0.3,0.3,0.1\rangle)
\end{array}\right\} \\
& G_{3}=\left\{\begin{array}{c}
s_{1}(\langle 0.1,0.4,0.3,0.2\rangle), s_{2}(\langle 0.4,0.1,0.3,0.2\rangle), s_{3}(\langle 0.3,0.2,0.4,0.1\rangle), \\
s_{4}(\langle 0.2,0.3,0.5,0.0\rangle), s_{5}(\langle 0.5,0.1,0.3,0.1\rangle)
\end{array}\right\} \\
& G_{4}=\left\{\begin{array}{c}
s_{1}(\langle 0.3,0.3,0.3,0.1\rangle), s_{2}(\langle 0.0,0.2,0.7,0.1\rangle), s_{3}(\langle 0.6,0.1,0.1,0.2\rangle), \\
s_{4}(\langle 0.4,0.4,0.2,0.0\rangle), s_{5}(\langle 0.1,0.5,0.3,0.1\rangle)
\end{array}\right\} \\
& G_{5}=\left\{\begin{array}{c}
s_{1}(\langle 0.5,0.1,0.3,0.1\rangle), s_{2}(\langle 0.3,0.4,0.3,0.0\rangle), s_{3}(\langle 0.6,0.1,0.3,0.0\rangle), \\
s_{4}(\langle 0.4,0.4,0.1,0.1\rangle), s_{5}(\langle 0.0,0.6,0.2,0.2\rangle)
\end{array}\right\}
\end{aligned}
$$

Discussion on Obtaining Results

Our purpose is to classify the patients $P_{j}(j=1,2,3)$ in one of the diagnoses classes $G_{i}(i=1,2,3,4,5)$. For this, the proposed picture fuzzy divergence measure has been measured from each $P$ to $G_{i}(i=1,2,3,4,5)$ and are given above. Results are shown in Table 1, picture fuzzy divergence measures assign the unknown patients class $P_{j}$ to the known diagnoses class as follows, Patients $P_{1}, P_{2}$ and $P_{3}$ falls in diagnoses $G_{1}, G_{5}$ and $G_{3}$, respectively, according to the principle of the minimum degree of divergence measure between PFSs.

Hence, conclude that the unknown class $P_{j}$ is related to the known class $G_{i}$ based on picture fuzzy divergence measure.

Table 1. Diagnosis Results for Picture Fuzzy Divergence Measure.

\begin{tabular}{cccccc}
\hline \multicolumn{5}{c}{ 1. $\left(\sigma_{\mathbf{1}}=\mathbf{0 . 1}, \sigma_{\mathbf{2}}=\mathbf{0 . 9}\right)$} \\
\hline$G_{\mathbf{1}}$ & $G_{\mathbf{2}}$ & $G_{\mathbf{3}}$ & $G_{\mathbf{4}}$ & $G_{\mathbf{5}}$ \\
\hline$P_{1}$ & $\mathbf{0 . 0 1 6 1 3}$ & 0.05002 & 0.05555 & 0.05219 & 0.04223 \\
$P_{2}$ & 0.06320 & 0.03644 & 0.04968 & 0.03881 & $\mathbf{0 . 0 3 5 3 7}$ \\
$P_{3}$ & 0.04012 & 0.07364 & $\mathbf{0 . 0 3 0 2 3}$ & 0.04423 & 0.05038 \\
\hline \multicolumn{5}{c}{ Results: $P_{1}\left(G_{1}\right), P_{2}\left(G_{5}\right), P_{3}\left(G_{3}\right)$} \\
\hline \multicolumn{5}{c}{ 2. $\left(\sigma_{\mathbf{1}}=\mathbf{0 . 2 ,} \sigma_{\mathbf{2}}=\mathbf{0 . 8}\right)$} \\
\hline$P_{1}$ & $\mathbf{0 . 0 2 8 6 6}$ & 0.08871 & 0.09853 & 0.09267 & 0.07496 \\
$P_{2}$ & 0.11445 & 0.06460 & 0.08818 & 0.06893 & $\mathbf{0 . 0 6 2 8 1}$ \\
$P_{3}$ & 0.07127 & 0.13060 & $\mathbf{0 . 0 5 3 6 9}$ & 0.07854 & 0.08943 \\
\hline \multicolumn{6}{c}{ Results: $P_{1}\left(G_{1}\right), P_{2}\left(G_{5}\right), P_{3}\left(G_{3}\right)$} \\
\hline \multicolumn{6}{c}{$G_{\mathbf{1}}$}
\end{tabular}


Table 1. Cont.

\begin{tabular}{|c|c|c|c|c|c|}
\hline \multicolumn{6}{|c|}{ 3. $\left(\sigma_{1}=0.3, \sigma_{2}=0.7\right)$} \\
\hline & $G_{1}$ & $G_{2}$ & $G_{3}$ & $G_{4}$ & $G_{5}$ \\
\hline$P_{1}$ & 0.03761 & 0.11623 & 0.12912 & 0.12153 & 0.09827 \\
\hline$P_{2}$ & 0.15383 & 0.08462 & 0.11561 & 0.09040 & 0.08238 \\
\hline$P_{3}$ & 0.09348 & 0.17110 & 0.07041 & 0.10301 & 0.11725 \\
\hline \multicolumn{6}{|c|}{ Results: $P_{1}\left(G_{1}\right), P_{2}\left(G_{5}\right), P_{3}\left(G_{3}\right)$} \\
\hline \multicolumn{6}{|c|}{ 4. $\left(\sigma_{1}=0.4, \sigma_{2}=0.6\right)$} \\
\hline & $G_{1}$ & $G_{2}$ & $G_{3}$ & $G_{4}$ & $G_{5}$ \\
\hline$P_{1}$ & 0.04297 & 0.13270 & 0.14743 & 0.13883 & 0.11222 \\
\hline$P_{2}$ & 0.18138 & 0.09659 & 0.13203 & 0.10327 & 0.09410 \\
\hline$P_{3}$ & 0.10680 & 0.19533 & 0.08043 & 0.11767 & 0.13391 \\
\hline \multicolumn{6}{|c|}{ Results: $P_{1}\left(G_{1}\right), P_{2}\left(G_{5}\right), P_{3}\left(G_{3}\right)$} \\
\hline \multicolumn{6}{|c|}{ 5. $\left(\sigma_{1}=0.5, \sigma_{2}=0.5\right)$} \\
\hline & $G_{1}$ & $G_{2}$ & $G_{3}$ & $G_{4}$ & $G_{5}$ \\
\hline$P_{1}$ & 0.04476 & 0.13818 & 0.15353 & 0.14459 & 0.11687 \\
\hline$P_{2}$ & 0.19710 & 0.10057 & 0.13750 & 0.10756 & 0.09801 \\
\hline$P_{3}$ & 0.11123 & 0.20340 & 0.08377 & 0.12255 & 0.13946 \\
\hline \multicolumn{6}{|c|}{ Results: $P_{1}\left(G_{1}\right), P_{2}\left(G_{5}\right), P_{3}\left(G_{3}\right)$} \\
\hline \multicolumn{6}{|c|}{ 6. $\left(\sigma_{1}=0.6, \sigma_{2}=0.4\right)$} \\
\hline & $G_{1}$ & $G_{2}$ & $G_{3}$ & $G_{4}$ & $G_{5}$ \\
\hline$P_{1}$ & 0.04297 & 0.13270 & 0.14743 & 0.13883 & 0.11222 \\
\hline$P_{2}$ & 0.20097 & 0.09659 & 0.13203 & 0.10327 & 0.09410 \\
\hline$P_{3}$ & 0.10680 & 0.19533 & 0.08043 & 0.11767 & 0.13391 \\
\hline \multicolumn{6}{|c|}{ Results: $P_{1}\left(G_{1}\right), P_{2}\left(G_{5}\right), P_{3}\left(G_{3}\right)$} \\
\hline \multicolumn{6}{|c|}{ 7. $\left(\sigma_{1}=0.7, \sigma_{2}=0.3\right)$} \\
\hline & $G_{1}$ & $G_{2}$ & $G_{3}$ & $G_{4}$ & $G_{5}$ \\
\hline$P_{1}$ & 0.03761 & 0.11623 & 0.12912 & 0.12153 & 0.09827 \\
\hline$P_{2}$ & 0.19292 & 0.08462 & 0.11561 & 0.09040 & 0.08238 \\
\hline$P_{3}$ & 0.09348 & 0.17110 & 0.07041 & 0.10301 & 0.11725 \\
\hline \multicolumn{6}{|c|}{ Results: $P_{1}\left(G_{1}\right), P_{2}\left(G_{5}\right), P_{3}\left(G_{3}\right)$} \\
\hline \multicolumn{6}{|c|}{ 8. $\left(\sigma_{1}=0.8, \sigma_{2}=0.2\right)$} \\
\hline & $G_{1}$ & $G_{2}$ & $G_{3}$ & $G_{4}$ & $G_{5}$ \\
\hline$P_{1}$ & 0.02866 & 0.08871 & 0.09853 & 0.09267 & 0.07496 \\
\hline$P_{2}$ & 0.17286 & 0.06460 & 0.08818 & 0.06893 & 0.06281 \\
\hline$P_{3}$ & 0.07127 & 0.13060 & 0.05369 & 0.07854 & 0.08943 \\
\hline \multicolumn{6}{|c|}{ Results: $P_{1}\left(G_{1}\right), P_{2}\left(G_{5}\right), P_{3}\left(G_{3}\right)$} \\
\hline \multicolumn{6}{|c|}{ 9. $\left(\sigma_{1}=0.9, \sigma_{2}=0.1\right)$} \\
\hline & $G_{1}$ & $G_{2}$ & $G_{3}$ & $G_{4}$ & $G_{5}$ \\
\hline$P_{1}$ & 0.01613 & 0.05002 & 0.05555 & 0.05219 & 0.04223 \\
\hline$P_{2}$ & 0.14065 & 0.03644 & 0.04968 & 0.03881 & 0.03537 \\
\hline$P_{3}$ & 0.04012 & 0.07364 & 0.03023 & 0.04423 & 0.05038 \\
\hline
\end{tabular}




\subsection{Divergence Measure for Pattern Recognition}

Assume that a set of known patterns $G_{i}(i=1,2,3)$ which are given in the PFSs form: Suppose the patterns $G_{i}(i=1,2,3)$ synthetic data information in universe set $S=\left\{s_{1}, s_{2}, s_{3}, s_{4}, s_{5}\right\}$ as

$$
\begin{aligned}
& G_{1}=\left\{\begin{array}{c}
s_{1}(\langle 0.4,0.1,0.3,0.2\rangle), s_{2}(\langle 0.5,0.2,0.3,0.0\rangle), s_{3}(\langle 0.4,0.0,0.3,0.3\rangle), \\
s_{4}(\langle 0.7,0.2,0.0,0.1\rangle), s_{5}(\langle 0.6,0.1,0.1,0.2\rangle)
\end{array}\right\} \\
& G_{2}=\left\{\begin{array}{c}
s_{1}(\langle 0.7,0.1,0.1,0.1\rangle), s_{2}(\langle 0.2,0.4,0.3,0.1\rangle), s_{3}(\langle 0.2,0.5,0.1,0.2\rangle), \\
s_{4}(\langle 0.1,0.2,0.5,0.2\rangle), s_{5}(\langle 0.3,0.3,0.3,0.1\rangle)
\end{array}\right\} \\
& G_{3}=\left\{\begin{array}{c}
s_{1}(\langle 0.1,0.4,0.3,0.2\rangle), s_{2}(\langle 0.4,0.1,0.3,0.2\rangle), s_{3}(\langle 0.3,0.2,0.4,0.1\rangle), \\
s_{4}(\langle 0.2,0.3,0.5,0.0\rangle), s_{5}(\langle 0.5,0.1,0.3,0.1\rangle)
\end{array}\right\}
\end{aligned}
$$

Consider an unknown pattern $A \in P F S$ is represented as

$$
A=\left\{\begin{array}{c}
s_{1}(\langle 0.6,0.1,0.2,0.1\rangle), s_{2}(\langle 0.3,0.2,0.4,0.1\rangle), s_{3}(\langle 0.4,0.2,0.3,0.1\rangle), \\
s_{4}(\langle 0.7,0.0,0.1,0.2\rangle), s_{5}(\langle 0.4,0.2,0.2,0.2\rangle)
\end{array}\right\}
$$

Discussion on Obtaining Results

In this study we classify the unknown pattern $A$ in one of the known patterns $G_{i}(i=1,2,3)$. For this, the proposed picture fuzzy divergence measure has from each $A$ to $G_{i}(i=1,2,3)$ and is given in Table 2. From the numerical results presented in the above table, picture fuzzy divergence measures assign the unknown patients class $A$ to the known class $G_{1}$, according to the principle of the minimum degree of divergence measure between PFSs.

Hence, conclude that the unknown class $A$ is related to the known class $G_{1}$ based on picture fuzzy divergence measure.

Table 2. Pattern Recognition Results for Picture Fuzzy Divergence Measure.

\begin{tabular}{cccc}
\hline$\widehat{\boldsymbol{P}_{\boldsymbol{F D}}}\left(\boldsymbol{A}, \boldsymbol{G}_{\boldsymbol{i}}\right)$ & $\widehat{\boldsymbol{P}_{\boldsymbol{F D}}}\left(\boldsymbol{A}, \boldsymbol{G}_{\mathbf{1}}\right)$ & $\widehat{\boldsymbol{P}_{\boldsymbol{F D}}}\left(\boldsymbol{A}, \boldsymbol{G}_{\mathbf{2}}\right)$ & $\widehat{\boldsymbol{P}_{\boldsymbol{F D}}}\left(\boldsymbol{A}, \boldsymbol{G}_{\mathbf{3}}\right)$ \\
\hline$\sigma_{1}=0.1, \sigma_{2}=0.9$ & $\mathbf{0 . 0 1 6 1 3 0 7}$ & 0.0500263 & 0.0555501 \\
$\sigma_{1}=0.2, \sigma_{2}=0.8$ & $\mathbf{0 . 0 2 8 6 6 4 9}$ & 0.0887184 & 0.0985386 \\
$\sigma_{1}=0.3, \sigma_{2}=0.7$ & $\mathbf{0 . 0 3 7 6 1 1 5}$ & 0.116239 & 0.129129 \\
$\sigma_{1}=0.4, \sigma_{2}=0.6$ & $\mathbf{0 . 0 4 2 9 7 6 8}$ & 0.132706 & 0.147436 \\
$\sigma_{1}=0.5, \sigma_{2}=0.5$ & $\mathbf{0 . 0 4 4 7 6 4 9}$ & 0.138187 & 0.153531 \\
$\sigma_{1}=0.6, \sigma_{2}=0.4$ & $\mathbf{0 . 0 4 2 9 7 6 8}$ & 0.132706 & 0.147436 \\
$\sigma_{1}=0.7, \sigma_{2}=0.3$ & $\mathbf{0 . 0 3 7 6 1 1 5}$ & 0.116239 & 0.129129 \\
$\sigma_{1}=0.8, \sigma_{2}=0.2$ & $\mathbf{0 . 0 2 8 6 6 4 9}$ & 0.0887184 & 0.0985386 \\
$\sigma_{1}=0.9, \sigma_{2}=0.1$ & $\mathbf{0 . 0 1 6 1 3 0 7}$ & 0.0500263 & 0.0555501 \\
\hline
\end{tabular}

\section{Advantages of the Proposed Picture Fuzzy Divergence Measure}

(1) Although to solve MADM problem in different areas, IFS theory has been profitably tested, some situations in real life IFSs are not applicable. In those situations we used the PFSs, which are an extension of IFSs, so the PFS is most often better than the IFS. In some situations, the IFS cannot solve a problem which PFS can solve, e.g., if a DM gives information about positive, neutral and negative membership grades, then this type of problem is only valid for the PFS. Particularly, PFS is more capable of handling uncertain problems.

(2) In this paper, we use the picture fuzzy information and proposed a technique to making decisions in complex real life problems. Numerical examples proposed in this paper cannot be handled with pre-existing structures such as the fuzzy sets, intuitionistic fuzzy sets and cubic sets. So, our proposed technique is a generalization of the pre-existing structure of fuzzy sets to deal with real life decision making problems more smoothly. 
(3) From the existing studies [8,16-18] it is observed that many researchers introduce different algorithms by utilizing divergence measures for IFSs. As mentioned above, in some situations, we cannot use the IFSs algorithm, because their corresponding algorithm may not give us applicable results.

(4) The divergence measures of IFS is a special case of the divergence measures of PFSs. Therefore, the defined picture fuzzy divergence measures are more generalized and acceptable to solve the MADM problem than the current ones.

\section{Conclusions}

In this article, we study and introduce an exponential Jensen picture fuzzy divergence measure successfully. The basic properties of picture fuzzy divergence measure are also introduced and discussed. Subsequently, we give a new method of decision-making problem based on the proposed divergence measure by analyzing the limitations and advantages in the existing literature. The decision steps of the decision method are also constructed. The proposed approach will yield an objective decision result based on information from the decision problem only. Some illustrative examples are used to show the appropriateness of the defined picture fuzzy divergence measure.

Author Contributions: Conceptualization, S.A. (Shahzaib Ashraf) and S.A. (Saleem Abdullah); methodology, S.A. (Shahzaib Ashraf); validation, S.Z.; formal analysis, S.Z.; writing-original draft preparation, S.A. (Shahzaib Ashraf); writing—review and editing, S.A. (Shahzaib Ashraf); supervision, S.A. (Saleem Abdullah).

Funding: This paper is supported by National Natural Science Foundation of China (No. 71671165), Zhejiang Province Natural Science Foundation (No. LY18G010007), Major Humanities and Social Sciences Research Projects in Zhejiang Universities (No. 2018QN058), Cooperation Project between Ningbo City and Chinese Academy of Social Sciences (No. NZKT201711) and K. C. Wong Magna Fund in Ningbo University.

Conflicts of Interest: The authors declare no conflict of interest.

\section{References}

1. Zadeh, L.A. Fuzzy sets. Inf. Control 1965, 8, 338-353. [CrossRef]

2. Atanassov, K.T. Intuitionistic fuzzy sets. Fuzzy Sets Syst. 1986, 20, 87-96. [CrossRef]

3. Yager, R.R. Pythagorean fuzzy subsets. In Proceedings of the Joint IFSA World Congress and NAFIPS Annual Meeting, Edmonton, AB, Canada, 24-28 June 2013; pp. 57-61.

4. Yager, R.R. Pythagorean membership grades in multicriteria decision making. IEEE Trans. Fuzzy Syst. 2014, 22, 958-965. [CrossRef]

5. Cuong, B.C. Picture Fuzzy Sets-First Results, Part 1. In Seminar "Neuro Fuzzy Systems With Applications"; Preprint 03/2013; Institute of Mathematics: Hanoi, Vietnam, 2013.

6. Cuong, B.C. Picture fuzzy sets. J. Comput. Sci. Cybern. 2014, 30, 409-420.

7. Son, L.H.; Thong, P.H. Some novel hybrid forecast methods based on picture fuzzy clustering for weather now casting from satellite image sequences. Appl. Intell. 2017, 46, 1-15. [CrossRef]

8. Son, L.H. Generalized picture distance measure and applications to picture fuzzy clustering. Appl. Soft Comput. 2016, 46, 284-295. [CrossRef]

9. Ashraf, S.; Mahmood, T.; Abdullah, S.; Khan, Q. Different approaches to multi-criteria group decision making problems for picture fuzzy environment. Bull. Braz. Math. Soc. New Ser. 2018. [CrossRef]

10. Garg, H. Some picture fuzzy aggregation operators and their applications to multi criteria decision-making. Arab. J. Sci. Eng. 2017, 42, 52-75. [CrossRef]

11. Phong, P.H.; Cuong, B.C. Multi-criteria group decision making with picture linguistic numbers. VNU J. Sci. Comput. Sci. Comput. Eng. 2017, 32, 39-53.

12. Wang, C.; Zhou, X.; Tu, H.; Tao, S. Some geometric aggregation operators based on picture fuzzy sets and their application in multiple attribute decision making. Ital. J. Pure Appl. Math. 2017, 37, 477-492.

13. Wei, G. Picture fuzzy hamacher aggregation operators and their application to multiple attribute decision making. Fund. Inform. 2018, 37, 271-320. [CrossRef]

14. Son, L.H.; Viet, P.V. Picture inference system: A new fuzzy inference system on picture fuzzy set. Appl. Intell. 2017, 46, 652-669. [CrossRef] 
15. Wei, G.; Alsaadi, F.E.; Hayat, T.; Alsaedi, A. Projection models for multiple attribute decision making with picture fuzzy information. Int. J. Mach. Learn Cybern. 2018, 9, 713-719. [CrossRef]

16. Joshi, R.; Kumar, S.; Gupta, D.; Kaur, H. A Jensen $\alpha$ norm dissimilarity measure for intuitionistic fuzzy sets and its applications in multiple attribute decision making. Int. J. Fuzzy Syst. 2017, 20, 1188-1220. [CrossRef]

17. Joshi, R.; Kumar, S. Exponential Jensen intuitionistic fuzzy divergence measure with applications in medical investigation and pattern recognition. Soft Comput. 2018. [CrossRef]

18. Srivastva, A.; Mahashwari, M. Decision making in medical investigations using new divergence measures for intuitionistic fuzzy sets. Iran J. Fuzzy Syst. 2016, 13, 25-44.

19. Garg, H.; Kaur, J. A Novel (R, S)-Norm Entropy Measure of Intuitionistic Fuzzy Sets and Its Applications in Multi-Attribute Decision-Making. Mathematics 2018, 6, 92. [CrossRef]

20. Ponta, L.; Carbone, A. Information measure for financial time series: Quantifying short-term market heterogeneity. Phys. A Stat. Mech. Appl. 2018, 510, 132-144. [CrossRef]

21. Pal, N.R.; Pal, S.K. Object background segmentation using new definition of entropy. IEE Proc. E 1989, 366, 284-295. [CrossRef]

22. Lin, J. Divergence measure based on Shannon entropy. IEEE Trans. Inf. Theory 1991, 37, 145-151. [CrossRef]

(C) 2019 by the authors. Licensee MDPI, Basel, Switzerland. This article is an open access article distributed under the terms and conditions of the Creative Commons Attribution (CC BY) license (http:/ / creativecommons.org/licenses/by/4.0/). 